\title{
Unveiling the magnetic nature of chromospheric vortices ${ }^{\star}$
}

\author{
Mariarita Murabito $^{1}$, Juie Shetye $e^{2,3}$, Marco Stangalini ${ }^{4}$, Erwin Verwichte ${ }^{2}$, Tony Arber $^{2}$, Ilaria Ermolli ${ }^{1}$, \\ Fabrizio Giorgi ${ }^{1}$, and Tom Goffrey ${ }^{2}$
}

\footnotetext{
1 INAF Istituto Nazionale di Astrofisica, Osservatorio Astronomico di Roma, 00078 Monte Porzio Catone, RM, Italy e-mail: mariarita.murabito@inaf.it

2 The Centre for Fusion, Space and Astrophysics, Department of Physics, University of Warwick, Coventry CV4 7AL, UK

3 Armagh Observatory and Planetarium, College Hill, Armagh BT61 9DB, UK

4 ASI - Agenzia Spaziale Italiana, Via del Politecnico snc, Rome, Italy
}

Received 5 May 2020 / Accepted 12 June 2020

\begin{abstract}
Context. Vortex structures in the Sun's chromosphere are believed to channel energy between different layers of the solar atmosphere. Aims. We investigate the nature and dynamics of two small-scale quiet-Sun rotating structures in the chromosphere.

Methods. We analysed two chromospheric structures that show clear rotational patterns in spectropolarimetric observations taken with the Interferometric Bidimensional Spectrometer at the Ca II $8542 \AA$ line.

Results. We present the detection of spectropolarimetric signals that manifest the magnetic nature of rotating structures in the chromosphere. Our observations show two long-lived structures of plasma that each rotate clockwise inside a $10 \operatorname{arcsec}^{2}$ quiet-Sun region. Their circular polarisation signals are five to ten times above the noise level. Line-of-sight Doppler velocity and horizontal velocity maps from the observations reveal clear plasma flows at and around the two structures. A magnetohydrodynamics simulation shows these two structures are plausibly magnetically connected. Wave analysis suggests that the observed rotational vortex pattern could be due to a combination of slow actual rotation and a faster azimuthal phase speed pattern of a magnetoacoustic mode.

Conclusions. Our results imply that the vortex structures observed in the Sun's chromosphere are magnetic in nature and that they can be connected locally through the chromosphere
\end{abstract}

Key words. Sun: chromosphere - Sun: magnetic fields - Sun: oscillations

\section{Introduction}

Rotating structures have been observed in both active- and quietSun regions of the solar atmosphere, and on a variety of spatial and temporal scales, since the beginning of the 20th century (see, for instance, Hale 1908). Terminologies such as vortices, tornados, or swirls have been employed to identify such rotating structures. For the sake of consistency, we shall call these structures vortices. Vortices of a few hundred kilometers in size have been identified across the solar atmosphere using modern telescopes supported by adaptive optics systems (e.g. Bonet et al. 2010; Attie et al. 2009; Vargas Domínguez et al. 2011; Wedemeyer-Böhm et al. 2012; Wedemeyer-Böhm \& Rouppe van der Voort 2009; Tziotziou et al. 2018). Quiet-Sun photospheric vortices have a typical size of $0.5-2 \mathrm{Mm}$ and a mean lifetime up to $15 \mathrm{~min}$ (Bonet et al. 2010; Wedemeyer et al. 2013; Liu et al. 2019a). There are estimated to be $3.1 \times 10^{-3}$ photospheric vortices $\mathrm{Mm}^{-2} \mathrm{~min}^{-1}$ (Bonet et al. 2010; Vargas Domínguez et al. 2011). Photospheric vortex flows are usually correlated with network magnetic elements at supergranular vertices (Requerey et al. 2018). In the quiet-Sun chromosphere, vortices have typical sizes of $1.5-4.4 \mathrm{Mm}$ and a lifetime of about $10-15 \mathrm{~min}$ up to $1.7 \mathrm{~h}$ (Wedemeyer-Böhm \& Rouppe van der Voort 2009; Tziotziou et al. 2018). They are seen to rotate as quasi-rigid bodies (Wedemeyer-Böhm et al. 2012; Tziotziou et al. 2018).

\footnotetext{
^ Movies are available at https://www . aanda.org
}

Numerical simulations have revealed that photospheric rotation forces the overlying plasma to rotate and lift up, and the magnetic field to twist (Wedemeyer-Böhm et al. 2012; Wedemeyer \& Steiner 2014; Yadav et al. 2020). Also, simulations show that rapidly changing photospheric perturbations propagate vertically along the magnetic field at the local Alfvén speed (Wedemeyer-Böhm et al. 2012; Shelyag et al. 2013; Liu et al. 2019b). It has been proposed that vortices are Alfvén waves that carry an average of $100-300 \mathrm{~W} \mathrm{~m}^{-2}$ Poynting flux into the upper atmosphere. These results support the findings on the quasi-periodic oscillations and short lifetimes of photospheric magnetic vortices reported from the simulations performed by Moll et al. (2011) and Kitiashvili et al. (2013). Vortices could also generate shocks and support magnetoacoustic waves (e.g. Fedun et al. 2011; Shelyag et al. 2013; Tziotziou et al. 2019). Shetye et al. (2019) show that chromospheric acoustic waves interact with vortices, leading to modified wave characteristics that may be used to probe vortex structure.

These studies are indicative of a magnetic nature of chromospheric vortices. However, direct observational evidence of the magnetic field of vortices in the upper layers of the solar atmosphere are still lacking. Extracting magnetic properties in the quiet-Sun chromosphere remains a challenge. Nevertheless, from the available chromospheric lines, the $\mathrm{Ca}$ II infrared triplet constitutes one of the best candidates for the analysis of chromospheric magnetic fields (Quintero Noda et al. 2016). In this paper we present the first spectropolarimetric measurements of two chromospheric rotating structures. 

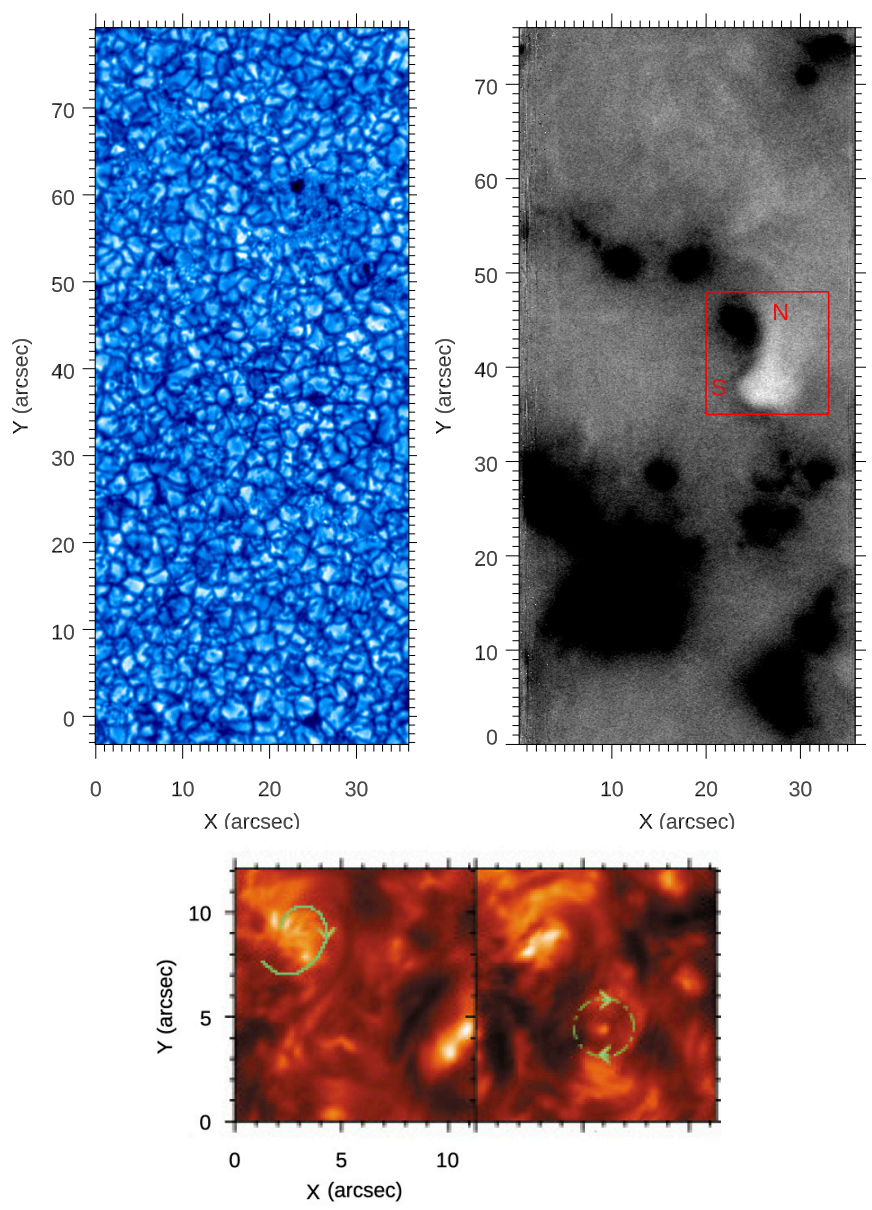

Fig. 1. Top left: white-light image at $6333 \AA$ of the IBIS FOV at 14:20 UT. Top right: mean CP map from IBIS Ca II $8542 \AA$ data. The signal in the CP map has been saturated to enhance contrast. Bottom: Ca II core snapshots with curves to emphasise the rotation pattern in the two structures, following the classification by Wedemeyer-Böhm \& Rouppe van der Voort (2009).

\section{Observational signatures}

We analysed observations of a quiet-Sun region acquired with the Interferometric Bidimensional Spectrometer (IBIS, Cavallini 2006) on May 1, 2015, from 14:18 UT to 15:03 UT, near disk centre. They consist of spectropolarimetric observations at a $46 \mathrm{~s}$ time cadence using photospheric Fe I $6173 \AA$ A nd chromospheric Ca II $8542 \AA$ lines sampled in 21 spectral points with a transmission of $20 \mathrm{~m} \AA$ and $60 \mathrm{~m} \AA$, respectively.

The field of view (FOV) is $40^{\prime \prime} \times 80^{\prime \prime}$ with a pixel scale of $0.08^{\prime \prime}$. Broadband white-light images with the same time cadence and FOV were taken at $6333.20 \pm 0.05 \AA$. In addition to the standard calibration procedures, the data were restored with multi-object multi-frame blind deconvolution (MOMFBD, van Noort et al. 2005). Although the polarisation signals detected in the chromosphere are low, the observations were taken under exceptional seeing conditions for the entire duration of the data sequence. This is also confirmed by the fact that the adaptive optics system was able to lock on the quiet-Sun, a challenging task for solar wavefront sensing. The exceptional conditions during observations render this dataset unique for data exploitation. We focussed on a $11.3^{\prime \prime} \times 12^{\prime \prime}$ region of interest $(\mathrm{ROI})$ that includes two rotating structures.
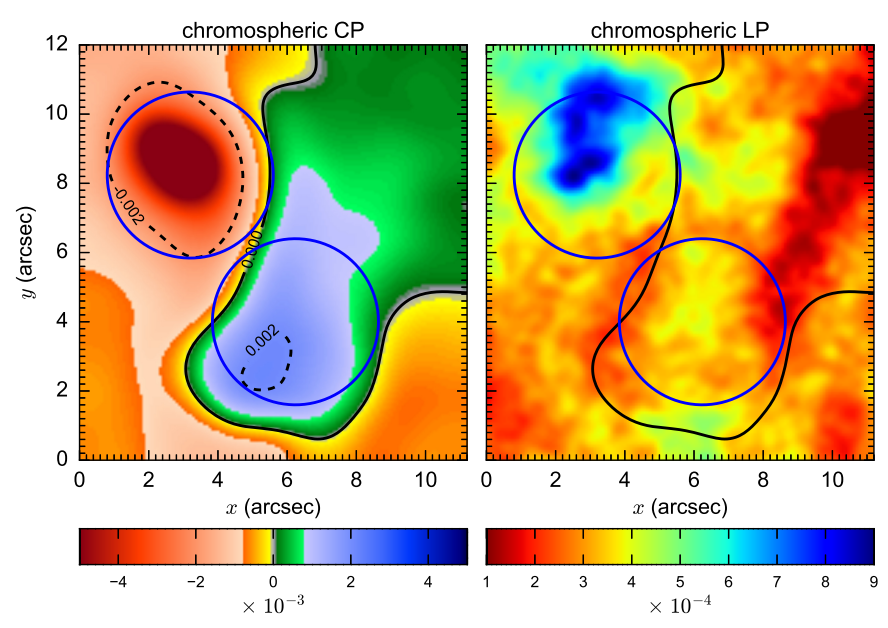

Fig. 2. Time-average CP (left) and LP maps (right). Contours indicate the polarity inversion line (solid line) and values of $\pm 2 \times 10^{-3}$ (dashed lines). Blue circles are centred on the $\mathrm{N}$ and $\mathrm{S}$ structures.

Figure 1 shows the white-light image and the map of mean chromospheric circular polarisation (CP) of the IBIS full FOV. When it is saturated to enhance relevant features, a clear pairing of opposite CP signals is revealed in the ROI. The CP is a measure of the line-of-sight (LOS) magnetic field, while linear polarisation (LP) is a measure of the inclined magnetic fields. We computed the CP and LP signals pixel-wise following the method set out by Martínez Pillet et al. (2011) and adapted to our specific data set as

$$
\left[\begin{array}{l}
\mathrm{LP} \\
\mathrm{CP}
\end{array}\right]=\frac{1}{10\left\langle I_{c}\right\rangle} \sum_{i=5}^{15}\left[\begin{array}{c}
\sqrt{Q_{i}^{2}+U_{i}^{2}} \\
\epsilon_{i}\left|V_{i}\right|
\end{array}\right], \epsilon_{i}= \begin{cases}1 & i<10 \\
0 & i=10 \\
-1 & i>10\end{cases}
$$

where $I_{i}, Q_{i}, U_{i}$, and $V_{i}$ are the Stokes parameters at line position $i$ and $\left\langle I_{c}\right\rangle$ is the average Stokes $I$ in the line-continuum in a quiet region within the FOV.

Figure 2, left panel, displays the time-average CP map, which indicates the presence of a magnetic dipole in the chromosphere separated by a curved polarity inversion line. Unfortunately, the $U$ and $Q$ Stokes signals that enter the LP calculation are too weak to extract meaningful information at each time step. Instead, we integrated the signal in time and produced an average LP map (see right panel of Fig. 2). It is likely that there are a horizontal components of the magnetic field in and around the studied structures.

The movie available online shows two structures at the location of this dipole. They are present during the entire observation period. For simplicity, we name the two structures North $(\mathrm{N})$ and South (S). They consist of small-scale bright and dark patches forming a pattern of spiral arms and concentric rings showing clockwise rotation. In particular, following the schematic classification introduced by Wedemeyer-Böhm \& Rouppe van der Voort (2009), we identify two different patterns in the Ca II line core maps (see bottom panel of Fig. 1). The S structure exhibits a roughly concentric ring pattern with a radius of about $1.5^{\prime \prime}$ during most of the observational time, while the $\mathrm{N}$ structure shows a spiral arm pattern.

The patches in $\mathrm{S}$ are clearest and rotate faster than in $\mathrm{N}$. For this reason, we identify $\mathrm{S}$ as a vortex but refer to $\mathrm{N}$ as a rotating "structure". The CP and LP signal (shown in Fig. 2) represent the first chromospheric spectropolarimetric measurement of a vortex. There is also a dark feature moving from the 

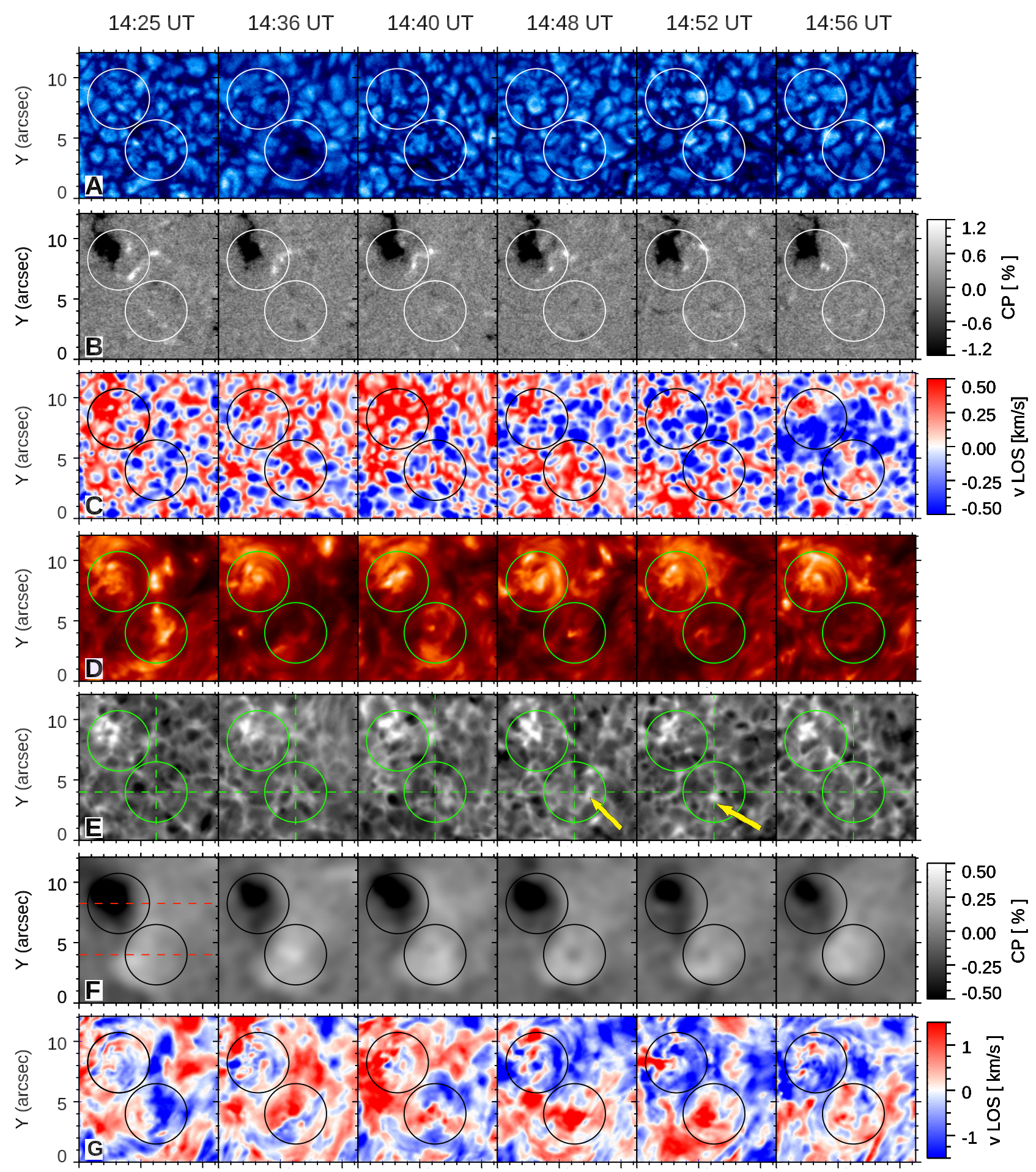

Fig. 3. Panels A-C: continuum intensity, CP maps, and LOS filtered velocity map from Fe I line measurements. Panels $D-G$ : line core intensity, line blue wing, CP maps, and LOS velocity maps from Ca II line measurements. Down-flow and up-flow correspond to positive and negative velocities, respectively. A Gaussian filter is applied to the chromospheric CP maps (panel $F$ ). The dashed horizontal lines indicate the slices where the time slice is computed (see Sect. 2 for more details). A movie of the data presented in this figure is available online.

south side of $\mathrm{N}$ to the east side of $\mathrm{S}$ along a curved trajectory. It wraps around $\mathrm{S}$ in the opposite direction of $\mathrm{S}$ rotation. There is a degree of uncertainty in flow tracking as seeing conditions, though excellent, may have affected contrast in regions of darkest intensity.

Figure 3 shows the evolution of the ROI in the photosphere (panels A-C) and the chromosphere (panels D-F) in more detail. In the photosphere, intensity images and $\mathrm{CP}$ maps show that the
$\mathrm{N}$ structure is anchored in an extended concentration of strong negative polarity magnetic flux. The $\mathrm{CP}$ signal in the $\mathrm{N}$ structure is larger than $1 \%$ (the noise level of the measurements is around $0.01 \%$ ). The $\mathrm{S}$ vortex lies above a patch of positive, spatiallydiffused magnetic flux. Unusual for a solar vortex, the $\mathrm{S}$ structure cannot be associated with a distinct photospheric flux concentration. Instead, it corresponds to a darker extended intergranular region. 


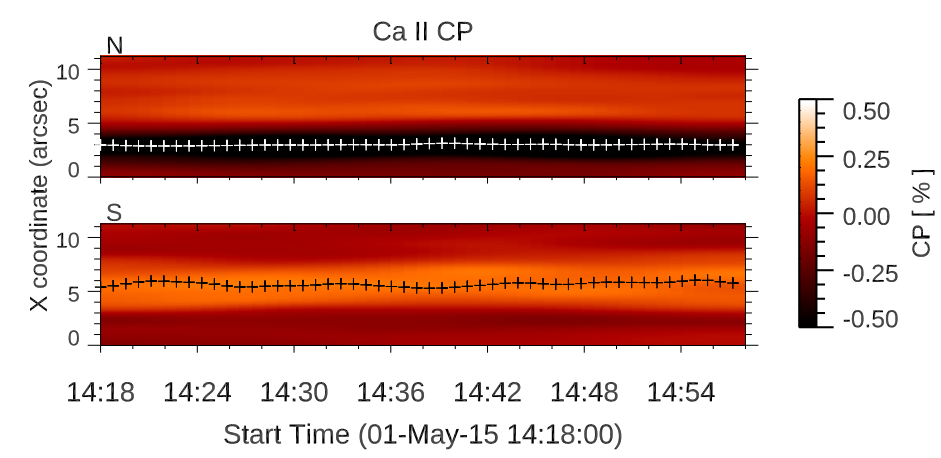

Fig. 4. Time-distance plot of the chromospheric circular polarisation along a cut at the centre of the two studied structures. The $x$-position of the barycentre of each structure is overplotted with a plus symbol. A Gaussian filter is applied to these plots.

The LOS velocity maps shown in Fig. 3 (panels $\mathrm{C}$ and E) have been constructed pixel-wise using a fit of Stokes $I$ with a linear background and a Gaussian line profile. The obtained velocities are band-pass filtered to remove $p$-modes. The photospheric LOS velocity map (see panels C) looks similar to the one expected from a pattern of granules, even if at 14:25 UT a strong red-shifted patch appears at the position of N, showing a down-flow at the magnetic structure.

The Ca II line core images (see panels D in Fig. 3) show two structures at the location of each of the two opposite-polarity flux concentrations consisting of bright and dark regions. Observations of the middle-upper photosphere sampled by the $\mathrm{Ca}$ line wing (see panels E) display small brightening events near the centre of the $\mathrm{S}$ structure (indicated by the yellow arrows). There are no such small events at the $\mathrm{N}$ structure. Instead, we detect a larger brightening region associated with the negative photospheric magnetic concentration.

Chromospheric CP maps (see panels F in Fig. 3) display a compact negative patch (with values larger than $0.5 \%$ and up to $1.5 \%$ ) that is co-spatial with $\mathrm{N}$ and a more diffused opposite polarity patch (with values up to $0.9 \%$ ) at $\mathrm{S}$. The $\mathrm{N}$ structure displays a mixture of up- and down-flow regions of the order of $0.5-0.7 \mathrm{~km} \mathrm{~s}^{-1}$ in chromosphere (see panels $\mathrm{G}$ in Fig. 3). The eastern side shows up-flows of about $0.5 \mathrm{~km} \mathrm{~s}^{-1}$. There is a persistent down-flow at the centre of the $S$ vortex (panels from $14: 36$ UT to $14: 52 \mathrm{UT}$ ) of $0.5 \pm 0.3 \mathrm{~km} \mathrm{~s}^{-1}$

Figure 4 shows time-distance plots of the $\mathrm{CP}$ signal along the two profiles indicated in the first panel of $\mathrm{CP}$ in Fig. 3. The $x$-position of their barycentre is annotated to better highlight the two structures. These values were computed by identifying each structure with a threshold in the CP. Both time-slice plots show that these structures are magnetically stable in time with a $\mathrm{CP}$ value higher than $0.5 \%$ and $0.2 \%$ for the $\mathrm{N}$ and $\mathrm{S}$ structures, respectively. It is noteworthy that the time-slice plot centered on $\mathrm{S}$ exhibits a negative polarity filament at around $X=3^{\prime \prime}$ (opposite to the positive polarity at the $\mathrm{S}$ vortex).

\subsection{Rotation}

To highlight the rotation of these structures in the chromosphere, we estimated the horizontal velocity field by applying an optical flow method that uses the two-frame motion estimation algorithm by Farneback (2003) to the Ca II line core data to obtain the displacement field at each time step. Image smoothing is employed to increase the robustness of the displacement field against noise, at the cost of losing some spatial resolution. The

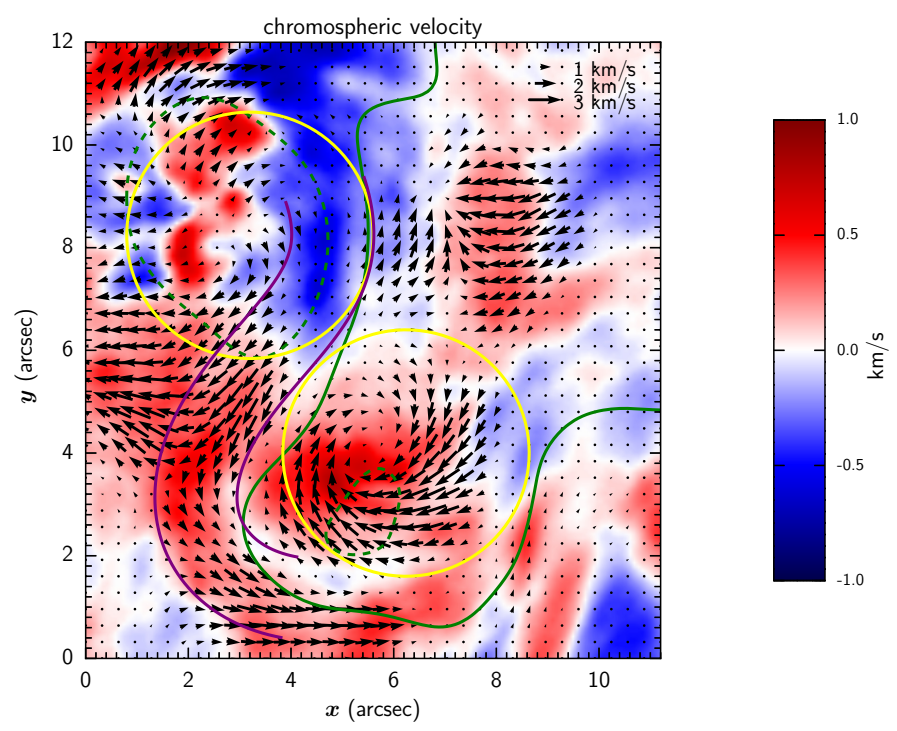

Fig. 5. Time-average chromospheric LOS velocity map with superimposed horizontal flow field. Green lines are contours of CP. The two purple lines outline the flow channel from $\mathrm{N}$ to $\mathrm{S}$.

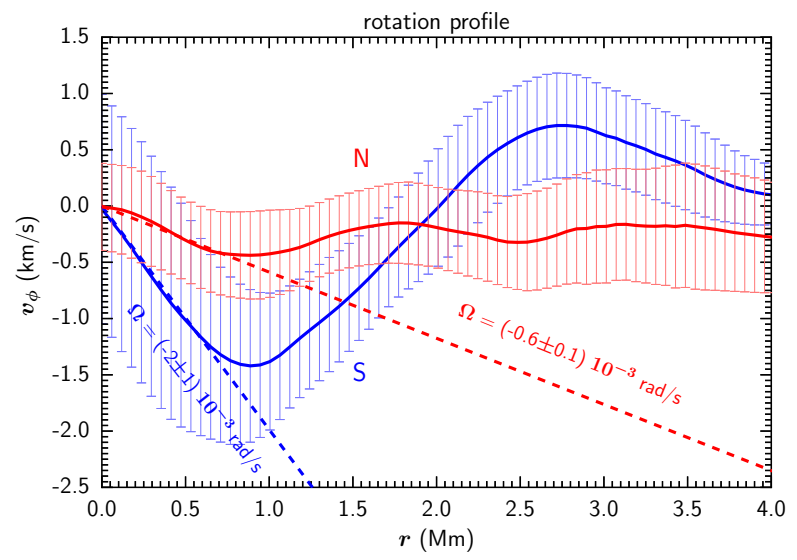

Fig. 6. Azimuthal (rotation) speed $v_{\phi}$ (solid lines) as a function of radius derived from the horizontal velocity in the polar grid centred on each structure averaged in time and over azimuthal angle. The dashed line is a linear fit to the first $0.7 \mathrm{Mm}$ of the profile.

average horizontal velocity field is derived from the mean of the displacement field across the whole observation period divided by the sequence time cadence. Figure 5 displays the mean chromospheric horizontal velocity map together with the LOS velocity. Both structures show a net clockwise horizontal rotation pattern. The $\mathrm{S}$ vortex's rotation pattern is the most obvious and stretches across $2 \mathrm{Mm}$.

We mapped the data onto a polar grid centred on each structure. This enables us to investigate each structure's local properties as a function of radial distance $r$ from its centre and as a function of azimuthal angle $\phi$.

Figure 6 shows the rotation profiles as a function of radius from the centre of each structure. Near the centre, the azimuthal speed $v_{\phi}$ is approximately negative and linear. This indicates rigid-body clockwise rotation with a constant negative angular velocity. The $\mathrm{N}$ structure rotates within a radius of $0.6 \mathrm{Mm}$ at an angular speed of $(-0.6 \pm 0.1) \times 10^{-3} \mathrm{rad} \mathrm{s}^{-1}$ (period of $\left.3 \mathrm{~h}\right)$. At larger radii $v_{\phi}$ remain negative but there is no discernible structure and there may not be a coherent rotation. 
The $\mathrm{S}$ rotation profile closely matches a rigid body rotation within a radius of about $1 \mathrm{Mm}$, with an angular speed of $(-2 \pm 1) \times 10^{-3} \mathrm{rad} \mathrm{s}^{-1}(50 \mathrm{~min})$. The azimuthal speed reaches a minimum value of $-1.5 \mathrm{~km} \mathrm{~s}^{-1}$ at $r=0.9 \mathrm{Mm}$. From $r=2 \mathrm{Mm}$ the sense of rotation reverses and becomes anticlockwise. This reflects the horizontal flow pattern visible in Fig. 5 that surrounds the $\mathrm{S}$ vortex to the east and south. There the LOS velocity has a similar magnitude and points downwards.

There is an extended region of negative LOS velocity at the western edge of $\mathrm{N}$ (see Fig. 5). This suggests that there is a connecting flow channel between the two structures, that is to say an up-flow emanating from near the western edge of $\mathrm{N}$ that then proceeds as a horizontal flow towards S. Around the S vortex the flow becomes downwards.

\subsection{Waves}

The mean velocity field rotation from optical flow is slower than visual estimates of rotation in Ca II intensity. We examined the dynamics as revealed by the spectrum of the polar maps in terms of radial and azimuthal wave numbers $k_{\mathrm{R}}$ and $m$, and angular frequency $\omega$ for a mode signal of the form $\exp \left[i\left(k_{\mathrm{R}} r+m \phi+\omega t\right)\right]$. The largest radius of the polar grids used are shown as circles in Fig. 5. Figure 7 shows the spectrum for the LOS velocity. The spectra for the $\mathrm{N}$ structure show oscillations at angular frequency $\omega_{0}= \pm 0.021 \mathrm{rad} \mathrm{s}^{-1} m=0$ and $k_{\mathrm{R}}=3.5 \mathrm{rad} \mathrm{s}^{-1}$. This corresponds to an azimuthally symmetric mode of the form $\cos \left(k_{\mathrm{R}} r-\omega t\right)$ that propagates radially outwards at a horizontal phase speed of $6.5 \mathrm{~km} \mathrm{~s}^{-1}$ with a periodicity of approximately five minutes. The spectra for the $S$ vortex show two clear peaks at five minutes with a mode symmetry of $\omega=-\omega_{0}, m=-1$ and $\omega=+\omega_{0}, m=+1$, which, when combined, is a mode of the form $\cos \left(m \phi+\omega_{0} t\right)$. This represents a five-minute oscillatory mode in the LOS velocity that propagates strictly clockwise in the plane of the sky. It has an $|m|=1$ azimuthal structure, that is to say at any given time each half of the vortex has a signal of opposite sign. A similar five-minute signal is present in intensity and CP.

To reveal the dynamics in each structure, we constructed time-distance plots for Ca II core intensity, LOS velocity, and $\mathrm{CP}$ from the polar maps (see the online movie of the ROI that has been band-pass filtered in time around $5 \mathrm{~min}$ ). We averaged over one spatial coordinate consistent with the mode propagation symmetry found in the spectral analysis in each structure. An overall mean is then subtracted to produce a perturbed signal. The CP perturbation is a proxy for the LOS magnetic field and magnetic pressure perturbations. The results are shown in Fig. 8. Following the spectral analysis, we averaged the signal in $\mathrm{N}$ across angle $\phi$ to focus on the wave propagation as a function of $r$ and time. The radially propagating waves are clear in intensity and LOS velocity, but not immediately visible in CP. We determined the speed of propagation from phase lag comparison between different radii and find it to be consistent with the spectral analysis.

For $\mathrm{S}$ we averaged the signal across the radius (up to $1.7 \mathrm{Mm}$ ) to examine wave propagation as a function of $\phi$ and time. The clockwise propagating five-minute mode with an $|m|=1$ azimuthal structure is visible in all signatures. At a radius of $1 \mathrm{Mm}$, the phase speed reaches $21 \mathrm{~km} \mathrm{~s}^{-1}$. Furthermore, crosswavelet analysis, as used in Shetye et al. (2019), reveals that the intensity perturbation leads the LOS velocity perturbation by a phase shift of approximately $90^{\circ}$, and is approximately in anti-phase with the $\mathrm{CP}$ perturbation. The signatures and phase relations are consistent with slow magnetoacoustic kink waves. The perturbations in CP may also be consistent with effects from

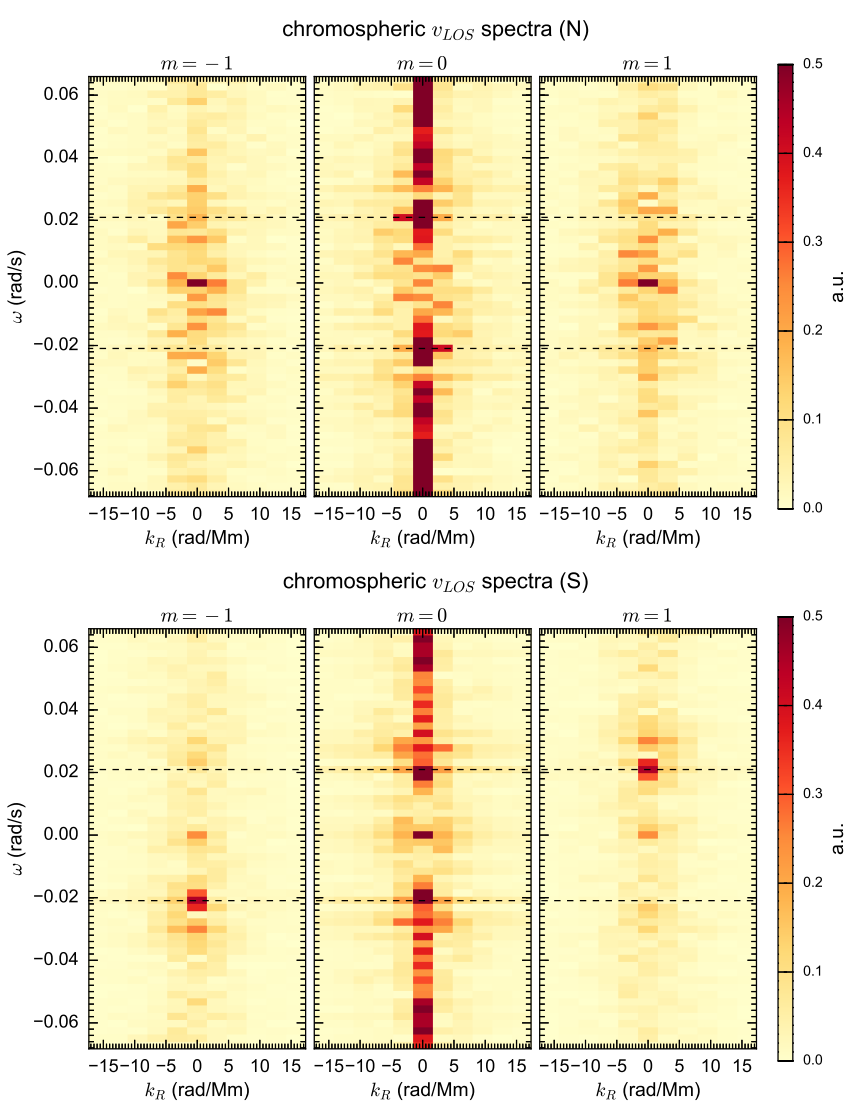

Fig. 7. Spectrum of $\mathrm{N}$ (top) and $\mathrm{S}$ (bottom) polar map in chromospheric LOS velocity as a function of radial wave number $k_{\mathrm{R}}$ and angular frequency $\omega$, for azimuthal wave number $m=-1$ (left), 0 (middle), 1 $($ right). The dashed lines indicate a signal with a five-minute periodicity.

opacity. Indeed, in the presence of a height dependence of the magnetic field (i.e. magnetic expansion), any fluctuation of the height of formation of the line may lead to spurious magnetic fluctuations which should not be considered as real magnetic oscillations. The CP contains variations with an $|m|=1$ structure on a longer timescale of approximately $25 \mathrm{~min}$. The slow rotation deduced from the optical flow analysis is not immediately visible here due to the dominance of the five-minute waves. But it is recovered if the waves are filtered out by smoothing over a longer timescale of about $10-15 \mathrm{~min}$.

\section{Discussion and conclusion}

We have reported unique spectropolarimetric measurements of two quiet-Sun chromospheric structures of opposite magnetic polarity, $\mathrm{N}$ and $\mathrm{S}$, showing clockwise rotation. These represent the first polarimetric signals of vortices in the chromosphere, and confirm what was previously only indirectly inferred. We reveal a clear pattern of circular polarisation signal in and around these two structures across the whole observational period. Although the linear polarisation signal at each single time step is weak, the time-integrated signal is well above the noise. The combined measurements of circular and linear polarisation patterns provide insight into the LOS and horizontal components of the magnetic field at the chromospheric level. In summary, polarimetry reveals the presence of two regions of opposite LOS magnetic field that are separated by a reverse S-shaped polarity inversion line where the magnetic field is predominantly horizontal. The negative LOS magnetic-flux region partially wraps itself 
A proofs:
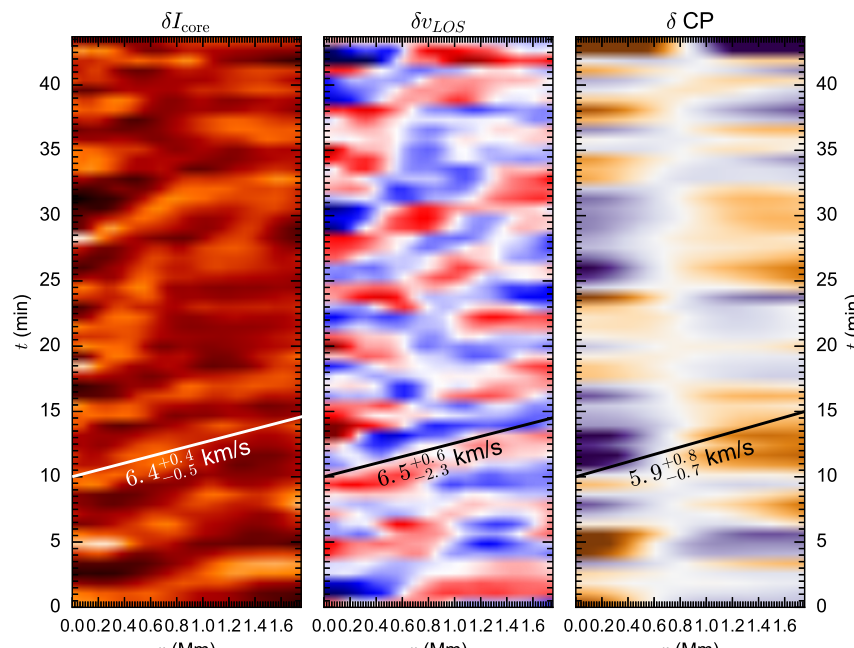

$(\mathrm{Mm})$

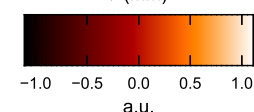

$r(\mathrm{Mm})$
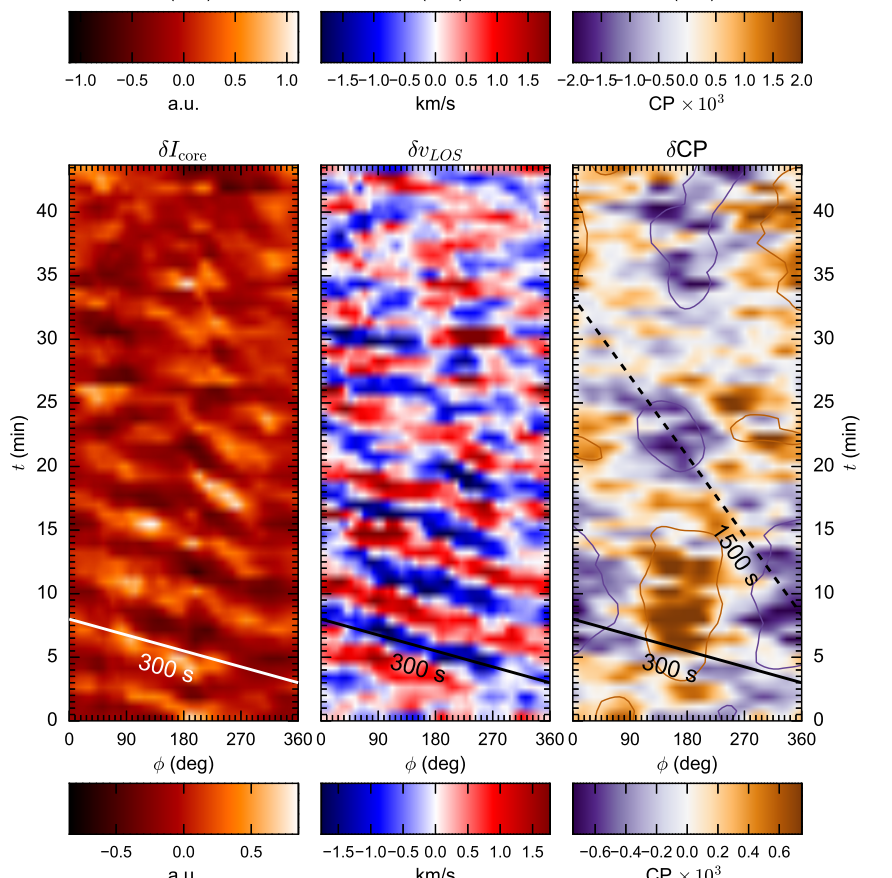
30
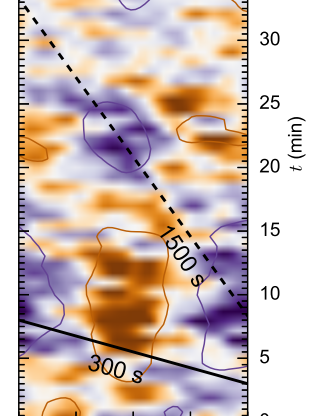

$90 \quad 180 \quad 270 \quad 360$ $\phi(\operatorname{deg})$
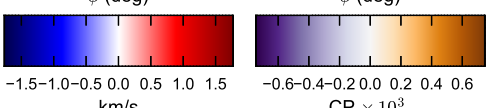

$\mathrm{km} / \mathrm{s}$

$\mathrm{CP} \times 10^{3}$

Fig. 8. Top panel: perturbations as a function of radial distance and time in intensity (left), LOS Doppler velocity (middle), and $\mathrm{CP}$ (right) in $\mathrm{Ca}$ II in a polar grid centered on $\mathrm{N}$ and averaged over azimuthal angle. The line highlights the phase propagation of the five-minute wave pattern with the speed from lag analysis. Bottom panel: perturbations in intensity (left), LOS Doppler velocity (middle), and CP (right) as a function of angle and time in a polar grid centered on $\mathrm{S}$ and averaged over radius. The line highlights the phase propagation of the wave patterns with their corresponding periodicity. A movie of the data filtered around $5 \mathrm{~min}$ is available online.

around the positive-flux region and $\mathrm{S}$ vortex. This pattern could be consistent with a non-potential magnetic dipole where both structures are magnetically connected. There is also a suggestion of a plasma flow from $\mathrm{N}$ towards and around $\mathrm{S}$ that is co-spatial with the magnetic polarity inversion line. It is worth noting that the global picture of the $\mathrm{N}$ - and $\mathrm{S}$-linked structures in our observations resembles highly twisted and sheared sigmoid magnetic structures in the coronal active regions (Canfield et al. 1999). Such structures are usually linked to rotation in sunspots
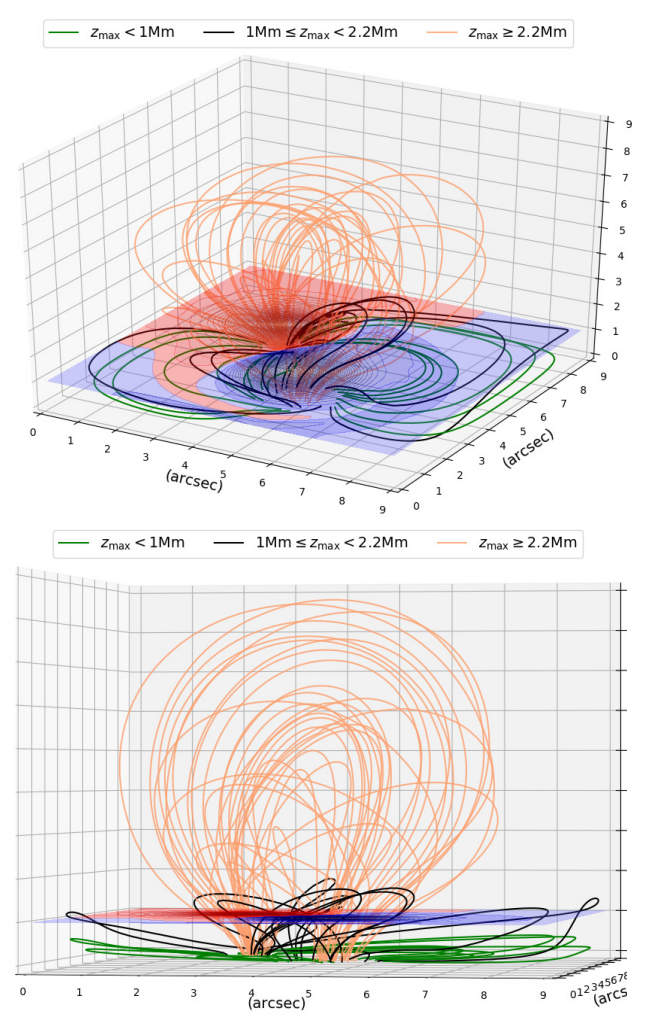

Fig. 9. 3D magnetic field configuration inferred from the 3D MHD simulation. The red- and blue-filled contours show the LOS magnetic flux at chromospheric height with red and blue representing positive and negative flux, respectively. A selection of magnetic field lines are traced.

(Tian \& Alexander 2006) indicating sheared and twisted magnetic field configurations (e.g. Jiang et al. 2014; Romano et al. 2014), but also in rotating network magnetic fields (Yan et al. 2015).

We reconstructed a candidate magnetic configuration of the two structures by using the Lare3d MHD code, which solves the non-linear magnetohydrodynamics (MHD) equations in three spatial dimensions (Arber et al. 2012) ${ }^{1}$. The configuration was initialized with the VAL-C (Vernazza et al. 1981) model atmosphere and a potential field. The exact location of the $S$ vortex flux is uncertain.

However, the observed photospheric CP shows clear evidence of a flux concentration below the $\mathrm{N}$, but no clear flux concentration below the $\mathrm{S}$. The chromospheric $\mathrm{CP}$ shows a clear dipolar nature. We assume that the photospheric source of the $\mathrm{S}$ vortex is composed of diffuse weak field. Therefore, we set up the simulations with a photospheric source of $1 \mathrm{kG}$ negative field with $0.5 \mathrm{Mm}$ radius at $\mathrm{N}$, and a larger area of weaker positive field, taken as $0.25 \mathrm{kG}$ with a radius of $1 \mathrm{Mm}$ at $\mathrm{S}$. The $\mathrm{S}$ flux source is then rotated slowly by $360^{\circ}$.

Figure 9 shows the converged three-dimensional field. It exhibits many features that are qualitatively in common with the spectropolarimetric observations presented above, that is to say distinctly shaped flux regions in the chromosphere that includes wrapping of positive flux around the $\mathrm{S}$ vortex, and a reverse S-shaped polarity inversion line with predominantly horizontal magnetic field between $\mathrm{N}$ and $\mathrm{S}$ sources. The horizontal

\footnotetext{
1 The current version of the code is available at the following link: https://warwick.ac.uk/fac/sci/physics/research/ cfsa/people/tda/larexd/
} 
field lines are co-spatial with the flow channel determined from the velocity analysis. The simulation underpins our conclusion that the two structures may be magnetically connected and that through their rotation this non-potential configuration is achieved. This simulation set-up will form the basis for future follow-up studies investigating rotation, flow, and wave propagation.

Previous studies have treated solar vortices in isolation or at least composed of individual substructures that form and reappear in and around vortex flow (Tziotziou et al. 2018). Opposite polarity flux sources associated with a vortex are often found close to each other (Wedemeyer-Böhm et al. 2012; Shetye et al. 2019; Liu et al. 2019c). Our observations show two clockwise rotating structures of opposite magnetic polarity over a field of view of only $10 \operatorname{arcsec}^{2}$. Our analysis of the spectropolarimetric signatures, flow patterns, and MHD simulation shows that the two structures can be magnetically connected locally through the chromosphere. This is of consequence for quantifying the transport of mass and energy into the corona through vortices. Unfortunately, our available observations do not allow us to investigate the formation of these two rotating structures as they appear to be already formed and stable at the beginning of the observation time.

The two structures rotate clockwise over the whole observational period, with lower rotation rates than those previously reported for chromospheric vortices (Wedemeyer-Böhm \& Rouppe van der Voort 2009; Tziotziou et al. 2018) but with a speed consistent with the rotation rates observed in photospheric vortices (Bonet et al. 2010; Vargas Domínguez et al. 2011). Near their centres the two structures rotate as a rigid body.s

Intensity, Doppler velocity, and CP in the chromosphere reveal five-minute waves similar to those reported from analysis of vortex motion by Shetye et al. (2019) and Tziotziou et al. (2019). At each structure these waves exhibit different local propagation characteristics. We interpret these local waves as slow magnetoacoustic in nature because their periodicity matches that found dominating throughout the FOV, have significant LOS velocity and intensity signatures, have consistent phase relations, and lack oscillatory behaviour in the azimuthal flow. The N structure carries (in projection) radially outward propagating waves. The waves in the $\mathrm{S}$ vortex propagate azimuthally with an $m=1$ phase speed pattern. Resonant absorption of sound waves by a magnetic flux tube favours the $|m|=1$ mode if the tube is thinner than the wavelength (Sakurai et al. 1991). The preference for clockwise propagation is indicative of an azimuthal asymmetry, such as magnetic twist or rotation.

We infer that the clockwise rotational $\mathrm{S}$ vortex pattern is due to a combination of rotational mass flow up to $1.5 \mathrm{~km} \mathrm{~s}^{-1}$ and an $|m|=1$ slow magnetoacoustic mode with a period of approximately $300 \mathrm{~s}$ and a phase speed of $20 \mathrm{~km} \mathrm{~s}^{-1}$ at the vortex edge. The flow would cause a Doppler shift in the mode of about $25 \mathrm{~s}$, but this cannot be discerned with the time cadence of $46 \mathrm{~s}$.

In general, chromospheric vortices may exhibit a superposition of fast rotational phase patterns due to MHD waves on top of a slower motion, in turn due to actual rotation (Tziotziou et al. 2019; Shetye et al. 2019). Careful wave analysis will be essential to distinguish steady rotation, wave-phase speed, and wave amplitude to obtain accurate measurements of the Poynting flux into the solar corona from MHD waves associated with vortices.
A comprehensive survey of the overall magnetic field topology of solar vortices is needed to assess the channelling of energy to higher atmospheric layers. Chromospheric spectropolarimetric measurements will be essential. In this regard, four-metre class solar telescopes will enable new highersensitivity and higher-accuracy spectropolarimetric measurements than those analysed in the present study to possibly provide further observational evidence of these processes down to unprecedented spatial resolutions.

Acknowledgements. We thank the referee for the useful suggestions. The authors thank Doug Gilliam (NSO) and Michiel Van Noort (MPS) for their valuable support during the acquisition and MOMFBD processing of the data. The research leading to these results has received funding from the European Union's Horizon 2020 research and innovation programme under Grant Agreement No 824135 (SOLARNET), the Istituto Nazionale di Astrofisica (INAF) and the Italian MIUR-PRIN grant 2017 "Circumterrestrial Environment: Impact of Sun-Earth Interaction”. JS is funded by STFC Grant ST/P000320/1. JS would like to thank INAF for supported visits. This research has made use of the IBIS-A archive. MS would like to thank University of Warwick for supported visit. The authors wish to acknowledge scientific discussions with the Waves in the Lower Solar Atmosphere (WaLSA; www . WaLSA . team) team, which is supported by the Research Council of Norway (project number 262622), and The Royal Society through the award of funding to host the Theo Murphy Discussion Meeting "High resolution wave dynamics in the lower solar atmosphere" (grant Hooke18b/SCTM).

\section{References}

Arber, T. D., Longbottom, A. W., Gerrard, C. L., et al. 2012, Astrophysics Source Code Library [record ascl:1208. 015]

Attie, R., Innes, D. E., \& Potts, H. E. 2009, A\&A, 493, L13

Bonet, J. A., Márquez, I., Sánchez Almeida, J., et al. 2010, ApJ, 723, L139

Canfield, R. C., Hudson, H. S., \& McKenzie, D. E. 1999, Geophys. Rev. Lett., 26,627

Cavallini, F. 2006, Sol. Phys., 236, 415

Farneback, G. 2003, Scand. Conf. Image Anal., 2749, 363

Fedun, V., Shelyag, S., Verth, G., et al. 2011, Annal. Geophys., 29, 1029

Hale, G. E. 1908, ApJ, 28, 100

Jiang, C., Wu, S. T., Feng, X., et al. 2014, ApJ, 780, 55

Kitiashvili, I. N., Kosovichev, A. G., Lele, S. K., et al. 2013, ApJ, 770, 37

Liu, J., Nelson, C. J., \& Erdélyi, R. 2019a, ApJ, 872, 22

Liu, J., Nelson, C. J., Snow, B., et al. 2019b, Nat. Commun., 10, 3504

Liu, J., Carlsson, M., Nelson, C. J., et al. 2019c, A\&A, 632, A97

Martínez Pillet, V., Del Toro Iniesta, J. C., Álvarez-Herrero, A., et al. 2011, Sol. Phys., 268, 57

Moll, R., Cameron, R. H., \& Schüssler, M. 2011, A\&A, 533, A126

Quintero Noda, C., Shimizu, T., de la Cruz Rodríguez, J., et al. 2016, MNRAS, 459, 3363

Requerey, I. S., Cobo, B. R., Gošić, M., \& Bellot Rubio, L. R. 2018, A\&A, 610, A84

Romano, P., Zuccarello, F. P., Guglielmino, S. L., et al. 2014, ApJ, 794, 118

Sakurai, T., Goossens, M., \& Hollweg, J. V. 1991, Sol. Phys., 133, 247

Shelyag, S., Cally, P. S., Reid, A., et al. 2013, ApJ, 776, L4

Shetye, J., Verwichte, E., Stangalini, M., et al. 2019, ApJ, 881, 83

Tian, L., \& Alexander, D. 2006, Sol. Phys., 233, 29

Tziotziou, K., Tsiropoula, G., Kontogiannis, I., Scullion, E., \& Doyle, J. G. 2018, A\&A, 618, A51

Tziotziou, K., Tsiropoula, G., \& Kontogiannis, I. 2019, A\&A, 623, A160

van Noort, M., Rouppe van der Voort, L., \& Löfdahl, M. G. 2005, Sol. Phys., 228, 191

Vargas Domínguez, S., Palacios, J., Balmaceda, L., et al. 2011, MNRAS, 416, 148

Vernazza, J. E., Avrett, E. H., \& Loeser, R. 1981, ApJS, 45, 635

Wedemeyer, S., \& Steiner, O. 2014, PASJ, 66, S10

Wedemeyer-Böhm, S., \& Rouppe van der Voort, L. 2009, A\&A, 507, L9

Wedemeyer-Böhm, S., Scullion, E., Steiner, O., et al. 2012, Nature, 486, 505

Wedemeyer, S., Scullion, E., Rouppe van der Voort, L., Bosnjak, A., \& Antolin, P. 2013, ApJ, 774, 123

Yadav, N., Cameron, R. H., \& Solanki, S. K. 2020, ApJ, 894, L17

Yan, X. L., Xue, Z. K., Pan, G. M., et al. 2015, ApJS, 219, 17 